\title{
Fiber optic sensor based on fluorescence quenching for heavy metal detection
}

\author{
Yadira A. Fuentes-Rubio ${ }^{1}$, René F. Domínguez-Cruz ${ }^{1, a}$, Oscar Baldovino-Pantaleón ${ }^{1}$, Carlos Ruiz-Zamarreño ${ }^{2, b}$ and \\ Francisco J. Arregui ${ }^{2}$ \\ (1) Electrical and Electronics Department. Autonomous University of Tamaulipas. Reynosa, Tamaulipas, México. \\ (2) Sensors Group. Department of Electrical and Electronic Engineering, Public University of Navarra, Pamplona, Spain. \\ arfdominguez@docentes.uat.edu.mx \\ b carlos.ruiz@upna.es
}

\begin{abstract}
In this paper, we present the preliminary results of a fiber optic sensor based on fluorescence quenching induced by mercury $(\mathrm{Hg})$ concentrations presence in aqueous solutions. The fabrication of the sensor head consists of a methyl red coating over multimode fiber tip using thermoplastic polyurethane to immobilize the thin layer. The sensor device was tested in a $\mathbf{H g}$ molar concentration ranges $10-10,000 \mathrm{nmol} / \mathrm{L}$. The experimental results show a linear dependence of the induced fluorescence quenching related to the $\mathrm{Hg}$ molar concentration.
\end{abstract}

Keywords-heavy metal, mercury, fluorescence quenching, fiber optic, multimode fiber, sensor

\section{INTRODUCTION}

Mercury $(\mathrm{Hg})$ is considered one of the most dangerous heavy metals and a priority issue for health organizations due to its toxicity, mobility, and a long time in the environment [1]. Once released into the atmosphere, $\mathrm{Hg}$ can be retained from 6 to 24 months in the surroundings. That would allow it to spread for thousands of kilometers in either the air, the water or the ground [1].

When the $\mathrm{Hg}$ is deposited in water, it can change its forms. Bacteria that live in aquifers can convert mercury to methylmercury [2]. Once turned, methylmercury can accumulate in organisms and contaminate the aquatic species. In consequence, the general population is potentially exposing to $\mathrm{Hg}$ through some foods. Harmful effects attributed to the consumption of foods contaminated with mercury has been reported. Neurodegenerative diseases in humans, such as Alzheimer's, Parkinson's, as well as altering the immune system and kidneys are examples of these dangerous consequences [4]. Therefore, the detection of the presence of $\mathrm{Hg}$ concentrations in water is a priority for the protection of human health [3].

In recent years, fiber optic-based heavy metal detection technologies using fluorescence effects have been proposed. For example, a miniature fiber optic sensor based on quantum dots (QD) has been reported for Hg detection [5]. This system allows detecting $\mathrm{Hg}^{2+}$ concentrations in the range of $1-500$ nmol/L. The sensor has a high sensitivity and response speed; however, the fabrication process is not easy, and it does not detect $\mathrm{Hg}$ concentrations below $1 \mathrm{nmol} / \mathrm{L}$. As a reference value, the maximum allowable level is $0.34 \mathrm{nmol} / \mathrm{L}$ for human safety, according to Directive 2013/39 of the European Union [6]. Also, surface plasmon resonance (SPR) in fiber using copper-silver nanoparticles coatings has been reported [7]. The proposed device allows to sense by quenching fluorescence a range of $0.01 \mu \mathrm{mol}$ to $1000 \mu \mathrm{mol}$ of $\mathrm{Hg}$ concentrations. Nevertheless, the construction of the sensor turns complex due to the preparation process of the nanoparticles and their subsequent integration into the $\mathrm{SiO} 2-\mathrm{TiO} 2-\mathrm{ZrO} 2$ matrix to coat the optical fiber.

In this paper, we report the preliminary data of a multimode fiber optic sensor coated with Methyl Red (MR) film using thermoplastic polyurethane to immobilize the thin layer. The principle of operation is based on induced fluorescence radiation in the MR film placed at the end of the MMF by a pump laser diode. When the tip is immersed in an aqueous solution with $\mathrm{Hg}$, we observe a fluorescence quenching effect as a function of $\mathrm{Hg}$ concentration. The reported device exhibits a detection range of $\mathrm{Hg}$ concentrations from $10 \mathrm{nmol} / \mathrm{L}$ to $10,000 \mathrm{nmol} / \mathrm{L}$. The sensor shows advantages such as easy construction, low cost and avoids additional measurement equipment.

\section{THEORY}

An attractive mechanism used for the detection of heavy metals is using fluorescence phenomena. For its implementation in optical waveguides [8], a fluorescent coating functionalized for an external agent is implanting on end (see upper scheme in Fig. 1). By propagating a pump beam, the generation of fluorescence emission is inducing and collected in the same channel. The coating area is sensitive to the external agent, which produces a change in the fluorescence signal through optical absorption called optical quenching (see the lower image in Fig. 1). In this process, the fluorescence intensity depends directly on the analyte concentration. This mechanism is how heavy metal ions can be recognized and detected by a fiber sensor device.

Fluorescence is an attractive optical effect for photonic sensors devices due to its high sensitivity and specificity [9]. The fluorescence intensity travel from the sensor head is generally proportional to the excitation light intensity and the

(C) 2020 IEEE. Personal use of this material is permitted. Permission from IEEE must be obtained for all other uses, in any current or future media, including reprinting/republishing this material for advertising or promotional purposes, creating new collective works, for resale or redistribution to servers or lists, or reuse of any copyrighted component of this work in other work. 
fluorophore concentration. However, this relationship is affected by quenching phenomena induced by external media.

The Stern-Volmer equation directly relates the intensity of emission in the absence $\left(I_{o}\right)$ and presence $\left(I_{Q}\right)$ of the quencher $(Q)$ in the sample [9] given as:

$$
\frac{I_{O}}{I_{Q}}=1+K_{S V} Q
$$

Where $K_{s v}$ is the Stern-Volmer constant $\left(K_{s v}=\tau_{o} k_{q}, k_{q}\right.$ is the bimolecular deactivation rate constant and $\tau_{o}$ is the duration of the excited state). Quenching data are usually presented as plots of $I_{O} / I_{Q}$ versus $Q$, which provide information about the concentration and quencher properties [10]. In our reported experiments, $\mathrm{Hg}$ particles take the role of the quencher element.

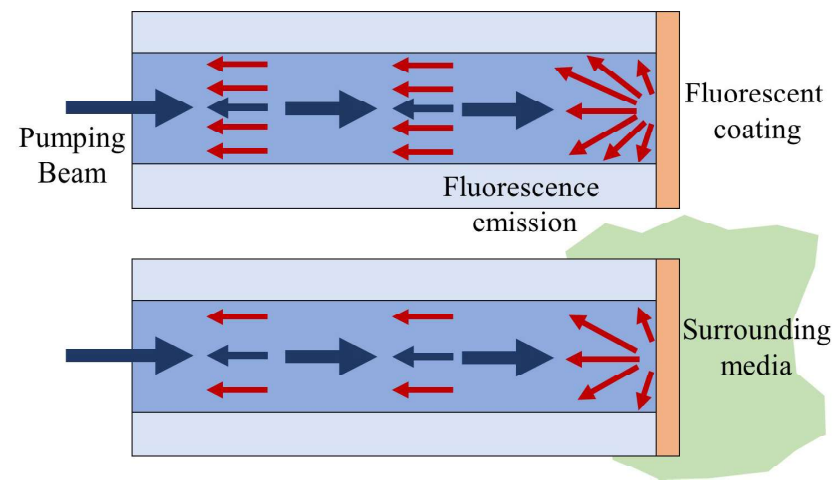

Fig. 1. A fluorescent coating is in a fiber optic tip. This coating is functionalizing for a specific external agent.

\section{EXPERIMENTAL}

\section{A. Sensor Head Fabrication}

Methyl red (MR) is a well-known acid-base indicator and has been using as a luminescent material for the detection of heavy metals [11]. MR shows fluorescence emission in the range $320 \mathrm{~nm}$ to $480 \mathrm{~nm}$ [12], with an emission maximum at $375 \mathrm{~nm}$ under excitation at $310 \mathrm{~nm}$. When MR is in contact with the $\mathrm{Hg}+$ ions, an absorption peak appears in the fluorescence signal.

The coating was prepared using $2.5 \mathrm{gr}$ MR indicator dissolved in $100 \mathrm{ml}$ ethanol, both provided by Sigma Aldrich ${ }^{\circledR}$. To immobilize MR on the fiber tip, we use 3.6gr thermoplastic polyurethane (TPU), Tecoflex $\AA$, provided by Lubrizol ${ }^{\circledR}$. The mixture was stirred for 3 hours at $60^{\circ} \mathrm{C}$ until obtaining a homogeneous solution. Additionally, the $\mathrm{pH}$ of the mixture was adjusted to 7 .

We use a segment of multimode fiber (Newport) with 125 $\mu \mathrm{m}$ as an external diameter and $62.5 \mu \mathrm{m}$ in core diameter. The MMF polymer was removed and cleaved fiber tip. Later, the fiber tip was repeatedly immersed in this solution using a dipcoating system (Nadatech ${ }^{\circledR}$ ), with a speed of $600 \mathrm{~mm} / \mathrm{min}$. The temperature was maintained at $60^{\circ} \mathrm{C}$ during all the dip-coating process. Then, the sensor head was placed into an oven for thermal fixing at $80^{\circ} \mathrm{C}$ for $15 \mathrm{~min}$ to evaporate the remaining solvent from the coating [11]. In Fig. 2, we show the final sensor device.

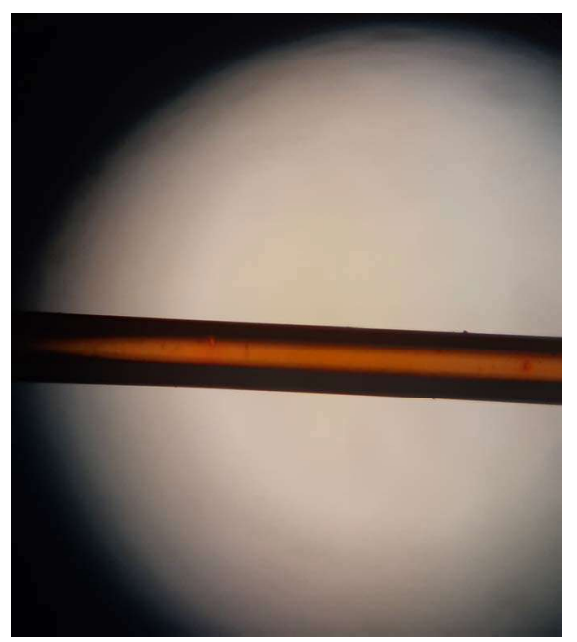

Fig. 2. Image of sensor built using MMF with MR coating.

\section{B. Hg metal ions concentrations}

According to Directive 2013/39 of the European Union, the maximum permissible concentration of $\mathrm{Hg}$ for the human organism is $0.34 \mathrm{nmol} / \mathrm{L}$ [6]. Besides, the Official Mexican Standard indicates that the maximum $\mathrm{Hg}$ presence is 4.9 $\mathrm{nmol} / \mathrm{L}$ [13]. Based on this standard, we prepare $\mathrm{Hg}$ concentrations $10^{-8}, 10^{-7}, 10^{-6}$ and $10^{-5} \mathrm{~mol} / \mathrm{L}$ using buffer solution with $\mathrm{pH} 7$ provided by PanReac ${ }^{\circledR}$ and a Mercury Stander Solution $\mathrm{Hg}$ provided by Merck ${ }^{\circledR}$.

\section{Experimental set-up}

The experiments were carried out using a LED Pyrois ${ }^{\circledR}$ as an optical pumping source (wavelength peak @ 365nm), which is pigtailed by multimode SMA cables (see Fig. 3). A $3 \mathrm{~dB}$ coupler was plugged on the system. The pumping field is launched within the coupler (port 1) until it reaches the sensor head, where it stimulates fluorescence in the MR coating (port 3 ). The induced optical field travels back through the same channel and is collected at port 2 by the Ocean Optics USB2000 + XR1-ES spectrometer and stored using a personal computer. An index matching gel is placed at port 4 to avoid unnecessary reflections.

Fig. 4 shows the spectrum of the LED pumping source centered at $365 \mathrm{~nm}$ (black line), which is obtained by connecting directly to the spectrometer. Also, the fluorescence beam detected in port 2 (red line) is showing, which is obtaining when the sensor head located in the air. Comparing both optical signals, we observed an additional peak corresponding to the fluorescent emission spectrum of the sensor built. This behavior is consistent with the well-known emission bandwidth of MR [12]. 


\section{RESULTS}

\section{A. Sensor head response to $\mathrm{Hg}$}

In order to know the sensor response in the presence of different concentrations of $\mathrm{Hg}$, the fiber optics tip was immersed in different aqueous samples. We started by dipping the sensor head in the lowest level of $\mathrm{Hg}\left(1 \times 10^{-8} \mathrm{~mol} / \mathrm{L}\right)$, later $1 \times 10^{-7} \mathrm{~mol} / \mathrm{L}$ and so on until reaching $1 \times 10^{-5} \mathrm{~mol} / \mathrm{L}$. These curves are showing in Fig. 5. It is important to note that for visualization purposes, all the traces are normalized. As we observe, a progressive fluorescence quenching in MR coating is due to different $\mathrm{Hg}$ concentrations. This effect is noted in the fluorescence peak is attenuating when the $\mathrm{Hg}$ level is growing (highlighted in the dotted line, Fig. 5).

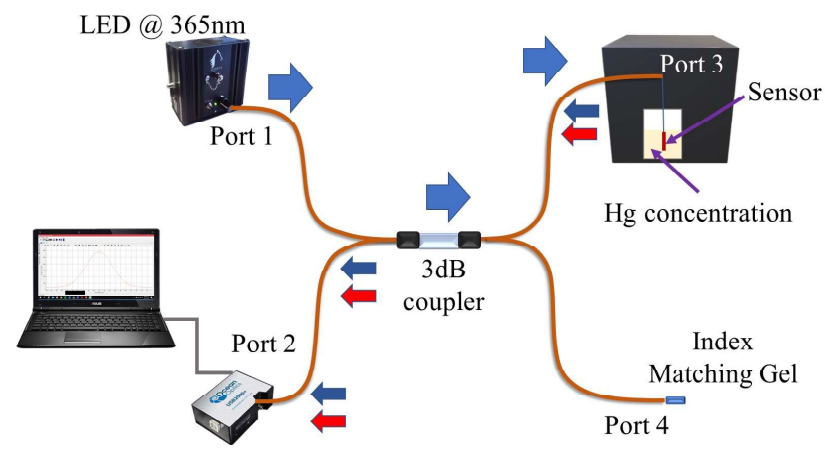

Fig. 3. Experimental set-up performed (arrow blue - pumping signal; arrow red - fluorescence signal).

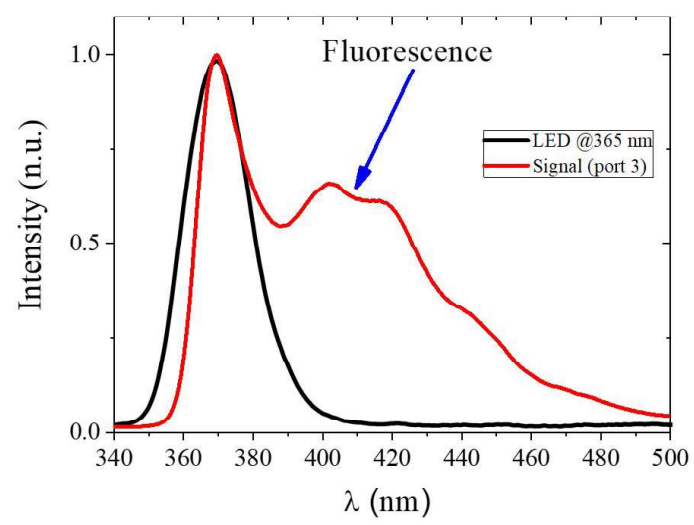

Fig. 4. Fluorescence signal detected at port 2 by the sensor head located in port 3 (red line). The fluorescence peak appears around $405 \mathrm{~nm}$. As a reference, the pumping source is sketching in the black line.

However, for a better description of the fluorescence decrement, we incorporate Stern-Volmer graph-based in equation 1 (Fig. 6). This analysis is obtained from the experimental data of each curve, specifically, the maximum fluorescence peak and the maximum intensity of the pumping beam. The calculation shows that they follow a sublinear dependence between rate $I_{O} / I_{Q}$ and concentration levels. When the $\mathrm{Hg}$ presence in buffer solution is increasing, the fluorescence quenching is also growing.

From Fig. 6, we observe one point does not maintain the linear behavior $\left(10^{-5} \mathrm{~mol} / \mathrm{L}\right)$. An incorrect adjustment in $\mathrm{pH}$ level would cause this mismatch. It is convenient to mention that in our experiments carried out, for high $\mathrm{Hg}$ concentration levels were difficult to keep its $\mathrm{pH}$ level over time, which is possibly regarding some chemical instability.

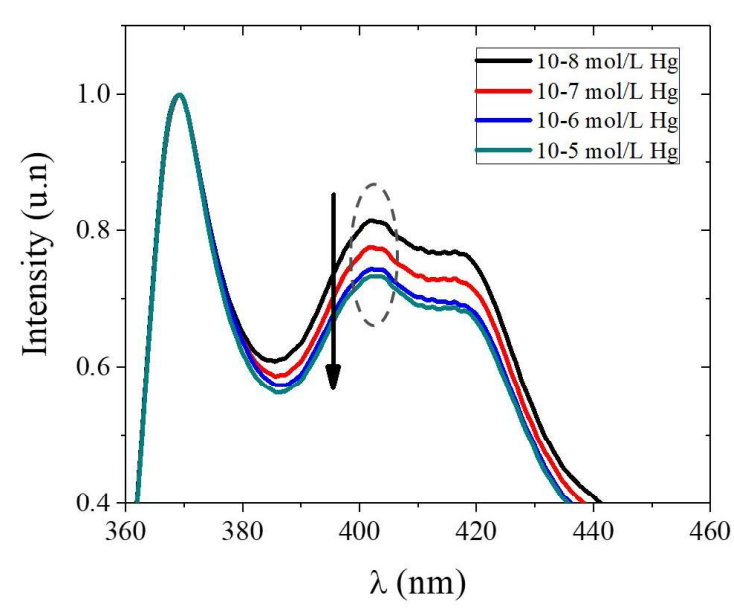

Fig. 5. The fluorescence signal for different levels of $\mathrm{Hg}$ concentration.

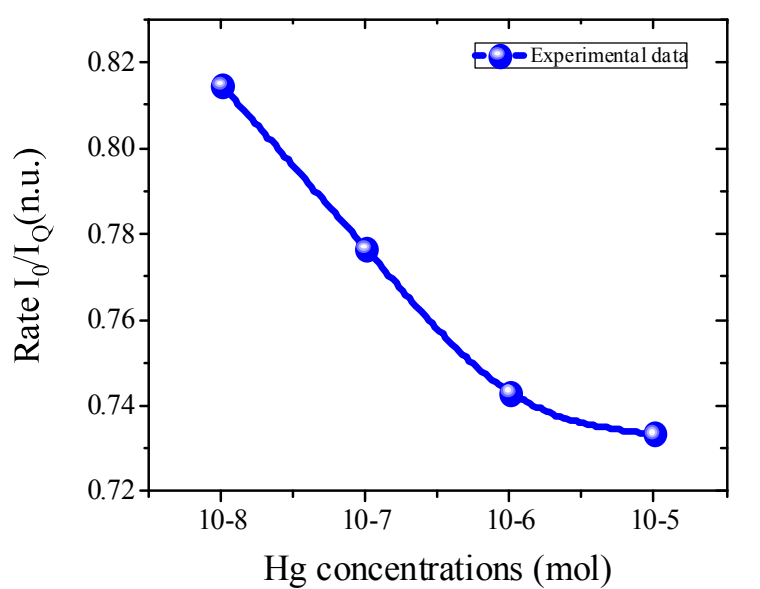

Fig. 6. Stern-Volmer trace based in experimental calculations generated by $\mathrm{Hg}$ contaminants.

\section{CONCLUSIONS}

In this paper we report the process of attaching a methyl red coating to a conventional multimode fiber tip applied as a novel sensor. The thin film was immobilized employing a thermoplastic polyurethane, which is placed by a conventional dip-coating technique. This system is capable to detect $\mathrm{Hg}$ concentrations in aqueous solutions in a reflection configuration. The operating principle of the sensor is based on fluorescence stimulation in the MR layer induced by a LED source. When the tip of the fiber is in contact with $\mathrm{Hg}$, a 
quenching of fluorescence is detected. The decrease in signal is dependent on $\mathrm{Hg}$ concentration in aquous solution. The optical device shown to operate in a molar concentration range of $\mathrm{Hg}$ $10^{-8}-10^{-5} \mathrm{~mol} / \mathrm{L}$. Also, the results indicate a sublinear response of the induced fluorescence quenching to the molar concentration of $\mathrm{Hg}$. This system performs with no additional complex systems, has low cost and simple manufacturing. As the next stage, we consider testing our device in a broader range of $\mathrm{Hg}$ concentrations and optimizing the construction process.

\section{ACKNOWLEDGMENTS}

This work was supported in part by the PRODEP-SEP under Grant UAT-CA-154 (IDCA-23742). Yadira A. FuentesRubio thanks CONACyT for the doctoral stay scholarship assigned.

\section{REFERENCES}

[1] J. X. Wang, X. B. Feng, C. W. N. Anderson, Y. Xing, L. H. Shang, "Remediation of mercury contaminated sites-A review". Journal of Hazard. Materials. Vol. 221-222, pp. 1-18, June 2012. doi: doi.org/10.1016/j.jhazmat.2012.04.035

[2] J. Duan, J. Zhan, "Recent developments on nanomaterials-based optical sensors for $\mathrm{Hg} 2+$ detection". Science China Press and Springer-Verlag Berlin Heidelberg. Vol. 58, pp.223-240. February 2015. doi: doi.org/10.1007/s40843-015-0031-8

[3] S. Ruan, H. Ebendorff-Heidepriem, Y. Ruan. "Optical fibre turn-on sensor for the detection of mercury based on immobilized fluorophore". Measurement. Vol. 121, pp. 122-126, June 2018. doi: doi.org/10.1016/j.measurement.2018.01.071

[4] J. Mutter, J. Naumann, C. Sadaghiani, H. Walach, G. Drasch, "Amalgam studies: disregarding basic principles of mercury toxicity", International Journal of Hygiene and Environmental Health. Vol. 207, pp. 391-397, January 2005. Doi: https://doi.org/10.1078/1438-463900305

[5] W. Wang, T. Liu and D. Yi, "Detection of mercury ion based on quantum dots using miniaturised optical fibre sensor," in The Journal of Engineering, Vol. 2019, no. 23, pp. 8595-8598, December 2019. doi: 10.1049 joe.2018.9063.

[6] "Directiva 2013/39/UE del Parlamento Europeo y del Consejo". Diario Oficial de la Unión Europea. 2013. [Online]. Available: https://www.boe.es/doue/2013/226/L00001-00017.pdf.

[7] V.P. Prakashan, G. George, M.S. Sanu, M.S. Sajna, A.C. Saritha, C. Sudarsanakumar, P.R. Biju, C. Joseph, N.V. Unnikrishnan, "Investigations on SPR induced $\mathrm{Cu} @ \mathrm{Ag}$ core shell doped SiO2-TiO2$\mathrm{ZrO} 2$ fiber optic sensor for mercury detection", Applied Surface Science. Vol.507, December 2019, doi: https://doi.org/10.1016/j.apsusc.2019.144957

[8] Y. N. Zhang, Y. Sun, L. Cai, Y. Gao, Y. Cai, "Optical fiber sensors for measurement of heavy metal ion concentration: A review", Measurement. Vol. 158, July 2020, doi: https://doi.org/10.1016/j.measurement.2020.107742

[9] C. Ruiz, F. Arregui, I. Matias, 2009. "Tesis Doctoral: Contribución Al Desarrollo De Sensores De Fibra Optica Basados En Recubrimientos Micro Y Nanoestructurados". Universidad Pública de Navarra, pp.7-9. 2009.

[10] J.R. Lakowicz. "Principles of Fluorescence Spectroscopy", 3rd. Ed. New York: Springer, 2016

[11] J. H. Wong, S. Lee. "pH-dependent fluorescence property of methyl red isomers in silver colloids", Physica B, Vol. 407, pp. 232-234, 2012. doi: 10.1016/j.physb.2011.10.036.

[12] K. D. Diganta, P. Goswami, Ch. B. Barman, B. Das. "Methyl red: A fluorescent sensor for $\mathrm{Hg} 2+$ over $\mathrm{Na}+, \mathrm{K}+, \mathrm{Ca} 2+, \mathrm{Mg} 2+, \mathrm{Zn} 2+$ and $\mathrm{Cd} 2+$." Environmental Engineering Research, Vol.17, pp.75-78, 2012. http://dx.doi.org/10.4491/eer.2012.17.S1.S75.

[13] Official Mexican Standard NOM-127-SSA1-1994, "Environmental Health, water for human use and consumption. Allowable limits of quality and treatments for its purification". Available: http://www.salud.gob.mx/unidades/cdi/nom/m127ssa14.html 